\title{
BITANGENTS OF NON-SMOOTH TROPICAL QUARTICS
}

\author{
HEEJONG LEE AND YOAV LEN
}

\begin{abstract}
We study bitangents of non-smooth tropical plane quartics. Our main result is that with appropriate multiplicities, every such curve has 7 equivalence classes of bitangent lines. Moreover, the multiplicity of bitangent lines varies continuously in families of tropical plane curves.
\end{abstract}

\section{INTRODUCTION}

The topic of bitangents to quartics curves has gained renewed attention recently with the introduction of tropical techniques. As shown in $\mathrm{BLM}^{+} 15$, every smooth tropical plane quartic admits seven families of bitangent lines. The relation between tropical and algebraic bitangents was later studied in [CJ15, JL16, LM17, Pan15]. The goal of the current note is to begin an examination of the bitangents when the curve is singular. In this case, the genus of the tropical curve may not be 3 anymore, and the number of bitangent lines may go down. However, there is a natural way of assigning a multiplicity to each of them (see Definition 4.1). Our main result is as follows.

Theorem (4.4). Let $\Gamma$ be a tropical plane quartic of genus $g$. Then $\Gamma$ has $2^{g}$ equivalence classes of bitangent lines. One of them has multiplicity $2^{3-g}-1$, and each of the others has multiplicity $2^{3-g}$.

When a bitangent has multiplicity 0 it means that it doesn't exist. In particular, when $g=3$, there are 7 bitangent lines with multiplicity 1 , and a single bitangent with multiplicity 0 , in accordance with $\left[\mathrm{BLM}^{+} 15\right.$, Theorem 3.9].

Non-hyperelliptic smooth algebraic curves of genus 3 are canonically embedded in the plane. Therefore, every line section of the curve gives rise to the canonical divisor, and their bitangents are in bijection with effective half canonical divisors. An analogous argument works for smooth tropical quartics. However, when the tropical curve is no longer smooth, and especially when its genus drops, this bijection falls apart. To overcome this difficulty, we parametrize the tropical quartic using a metric graph of genus 3, which we refer to as the paired metric graph, and obtain an upper bound on the number of bitangent lines.

On the other hand, to obtain a lower bound on the number of bitangents, we use a limiting process. Given a non-smooth quartic, we approximate it using a sequence of smooth quartics. Via upper semicontinuity of intersection numbers, each of the 7 bitangent lines of the smooth quartic converges in the limit to a bitangent of our non-smooth quartic.

Acknowledgements. This paper arose from the second author's project group at the 2017 Fields Undergraduate Summer Research Program funded by Fields Institute. We thank the Fields Institute for this prestigious opportunity. We wish to thank the other member of the group, Run Yu Tan, Akshay Tiwary, and Wanlong Zheng for insightful comments and fruitful discussions, and we thank Ralph Morrison and Dhruv Ranganathan for helpful comments and remarks. Finally, we thank the referees for their insightful remarks. 


\section{Preliminaries}

In this section, we provide a brief overview of some of the basic terms in tropical geometry that will be used throughout. See [MS15, Chapter 1] for more detail.

2.1. Tropical plane curves. A tropical plane curve $\Gamma$ is a planar metric graph possibly with unbounded edges, together with a weight function $w_{E}: E \rightarrow \mathbb{Z}_{>0}$, such that each edge has a rational slope, satisfying the following balancing condition: the weighted sum of the outgoing primitive integral vectors emanating from every vertex is zero. The last condition is called the balancing condition. For the rest of the paper, $\Gamma$ will denote a plane tropical curve.

Every tropical curve is dual to a regular subdivision of a lattice polygon, known as its subdivided Newton polygon. Each edge of the curve corresponds to an edge of the Newton polygon, and each vertex corresponds to a face. The weight of an edge is the lattice length of the corresponding edge of the polygon. Note that the Newton polygon determines the directions and weights of the edges of the tropical curve, but not the length.

Definition 2.1. A tropical curve $\Gamma$ has degree $d$ if its Newton polygon is a standard right triangle of edge length $d$.

In particular, a tropical quartic is dual to a right angled triangle of edge length 4 . Note that a general tropical line meets a tropical curve of degree $d$ at $d$ points. If the Newton polygon consists only of triangles with area $\frac{1}{2}$, then the curve is said to be smooth.

2.2. Singularities of tropical plane curves. Each vertex of $\Gamma$ corresponds to a face of the Newton polygon. The curve is said to be singular at $v$ if the area of the dual face is strictly greater than $\frac{1}{2}$. The following theorem determines precisely when that occurs [Pic99].

Theorem 2.2 (Pick's theorem). Let $P$ be a lattice polygon with area A. Then

$$
A=\frac{b}{2}+i-1
$$

where $b$ denotes the number of lattice points on the boundary and $i$ denotes the number of lattice points in the interior.

Since every polygon has at least three vertices, its area is greater than $\frac{1}{2}$ if and only if it has additional lattice points either on the boundary or in the interior. Figure 1 shows three different ways to obtain singularities. In the first case, the edge adjacent to the vertex has weight 2 . In the second case, the vertex is 4 -valent. The third type of singularity occurs when the face dual to the vertex contains a lattice point in its interior, which means that the genus of the entire curve has dropped. We keep track of the lost genus by adding integer weights on the vertices.
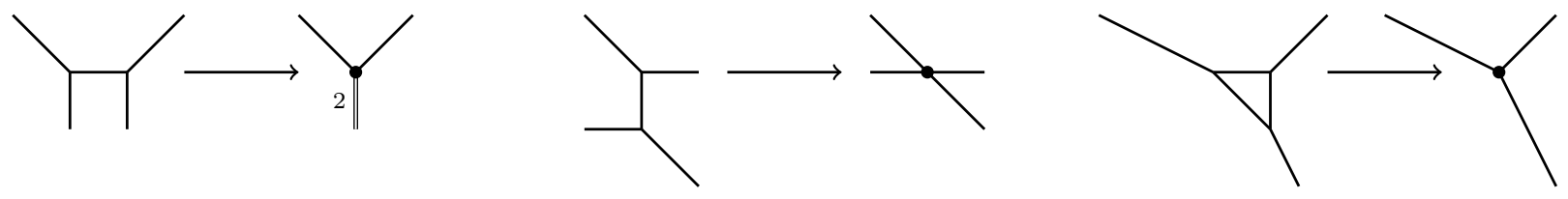

FiguRE 1. Singular vertices on tropical plane curve.

Definition 2.3. The weight function $w_{\Gamma}: \Gamma \rightarrow \mathbb{Z}_{\geq 0}$ assigns to each vertex of $\Gamma$ the number of lattice points in the interior of its dual face, and is zero everywhere else. 

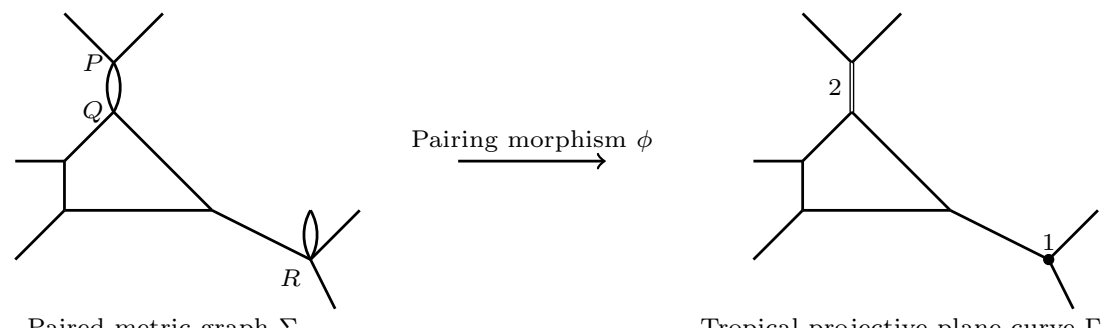

FIGURE 2. Paired metric graph and pairing morphism.

Given a tropical plane curve $\Gamma$, we parametrize it with a metric graph $\Sigma$ (possibly with infinite edges). The vertex set of $\Sigma$ is the same as $\Gamma$. Every edge of weight $w_{e}$ of $\Gamma$ is replaced with $w_{e}$ edges of $\Sigma$ of the same length. Whenever a vertex $v$ of $\Gamma$ has weight $w_{v}>0$, we attach $w_{v}$ loops of length $\epsilon>0$ based at the corresponding vertex of $\Sigma$. None of our results below depend on the length of these loops. We have a natural map $\phi: \Sigma \rightarrow \Gamma$ which collapses loops and multiple edges. See Figure 2 for an example.

Definition 2.4. The metric graph $\Sigma$ constructed this way is called the paired metric graph of $\Gamma$, and the map $\phi$ is called the pairing morphism.

It is straightforward to check that the genus $g_{\Sigma}$ of $\Sigma$ is exactly $\frac{(d-1)(d-2)}{2}$.

2.3. Divisor theory on metric graphs. In this section, we briefly recall the theory of divisors on metric graphs. A detailed discussion can be found in BJ16, GK08.

Let $\Sigma$ be a metric graph. The divisor group $\operatorname{Div}(\Sigma)$ is the free abelian group on the points of $\Sigma$. An element $D$ of $\operatorname{Div}(\Sigma)$ is called a divisor. Each divisor has a unique representation

$$
D=a_{1} \cdot p_{1}+a_{2} \cdot p_{2}+\cdots+a_{n} \cdot p_{n} .
$$

When the coefficient of $p$ at $D$ is $a$, we write $D(p)=a$, and say that $D$ has $a$ chips at $P$. The degree of $D$ is $\operatorname{deg}(D):=a_{1}+a_{2}+\cdots a_{n}$, and $D$ is said to be effective if all the coefficients are non-negative.

A rational function on $\Sigma$ is a continuous piecewise linear function with integer slopes. For each rational function $f$ on $\Sigma$, we associate a divisor $\operatorname{div}(f)$ where the coefficient at each point $p$ is equal to the sum of incoming slopes of $f$ at $p$. Such divisors are referred to as principal, and together they form a subgroup $\operatorname{Prin}(\Sigma)$ of $\operatorname{Div}(\Sigma)$. Divisors $D_{1}, D_{2}$, are said to be linearly equivalent if $D_{1}-D_{2}$ is principal. The quotient group $\operatorname{Pic}(\Sigma):=\operatorname{Div}(\Sigma) / \operatorname{Prin}(\Sigma)$ is called the Picard group of $\Sigma$. The restriction to divisors of degree zero is the Jacobian of $\Sigma$, denoted $\operatorname{Jac}(\Sigma)$.

A divisor $D$ has rank $r$ if $D-E$ is linearly equivalent to an effective divisor, for every effective divisor $E$ of degree $r$, and $r$ is the largest integer with this property. The canonical divisor is any divisor linearly equivalent to

$$
K_{\Sigma}:=\sum_{v \in \Sigma}(\operatorname{val}(v)-2) \cdot v
$$

By a double counting argument, the degree of the canonical divisor is $2 g-2$. From the tropical Riemann-Roch theorem [GK08, its rank is $g-1$.

Definition 2.5. A theta characterisctic of a metric graph $\Sigma$ is a divisor $\theta$ which satisfies $2 \theta \sim K_{\Sigma}$. 
2.4. Divisor theory on tropical plane curves. For a smooth tropical or algebraic curve, linear equivalence of divisors is closely related with intersections: the pullback of two different lines gives rise to equivalent divisors. However, this relation breaks when the curve is not smooth. Therefore, statements of interest to us will be stated and proved on the paired metric graph and shifted to the tropical plane curve.

Let $\Gamma$ be a tropical plane curve, and let $\phi: \Sigma \rightarrow \Gamma$ be the paired metric graph. As usual, Div $(\Gamma)$ is the free abelian group generated by the set of points of $\Gamma$. However, linear equivalence of divisors needs to be defined differently. The pairing morphism induces a group homomorphism $\phi_{*}$ from $\operatorname{Div}(\Sigma)$ to $\operatorname{Div}(\Gamma)$, by defining $\phi_{*}(D)(P)=\sum_{P^{\prime} \in \phi^{-1}(P)} D\left(P^{\prime}\right)$.

Definition 2.6. The Picard group of $\Gamma$ with respect to $\Sigma$ is

$$
\operatorname{Pic}(\Sigma, \Gamma):=\operatorname{Div}(\Gamma) / \phi_{*}(\operatorname{Prin}(\Sigma)) \text {. }
$$

Similarly, the Jacobian of $\Gamma$ with respect to $\Sigma$ is

$$
J(\Sigma, \Gamma):=\operatorname{Div}_{0}(\Gamma) / \phi_{*}(\operatorname{Prin}(\Sigma)) .
$$

One easily sees that $\operatorname{Pic}(\Sigma, \Gamma) \cong \operatorname{Pic}(\Sigma) /\left\langle[Q]-\left[Q^{\prime}\right]\right\rangle_{\phi(Q)=\phi\left(Q^{\prime}\right)}$.

The group $\operatorname{Pic}(\Sigma, \Gamma)$ can be identified as the usual Picard group of another metric graph. Let $\Gamma^{w}$ be the non-weighted metric graph obtained from $\Gamma$ by rescaling every edge $e$ by $\frac{1}{w_{e}}$ and forgetting the weights. Then there is a natural map between $\Gamma$ and $\Gamma^{w}$ given by rescaling edges accordingly, that is extended by linearity to a map on divisors.

Proposition 2.7. The Picard group of $\Gamma^{w}$ is isomorphic to the Picard group $\operatorname{Pic}(\Sigma, \Gamma)$.

Proof. It is clear that the map from $\Gamma$ to $\Gamma^{w}$ induces a bijection between $\operatorname{Div}(\Gamma)$ and $\operatorname{Div}\left(\Gamma^{w}\right)$. We need to show that principal divisors on $\Sigma$ give rise to principal divisor on $\Gamma^{w}$, and that every principal divisor arises this way. Let $D$ be a principal divisor on $\Sigma$ such that $D=\operatorname{div}(f)$ for some $f$, and let $D^{\prime}$ be the corresponding divisor on $\Gamma^{w}$. Define a function $f^{\prime}$ on $\Gamma^{w}$ whose slope at every point is the sum of the slopes at the corresponding points of $\Sigma$. Then $f^{\prime}$ defines a continuous piecewise linear function with $\operatorname{div}\left(f^{\prime}\right)=D^{\prime}$.

Conversely, given a principal divisor $E^{\prime}=\operatorname{div}\left(g^{\prime}\right)$ on $\Gamma^{w}$, we define a function $g$ on $\Sigma$ as follows. Let $e$ be an interval of length $\ell(e)$ where the slope of $g^{\prime}$ is $s$, contained in an edge $\Gamma^{w}$ of weight $k$. Then, by definition, there are $k$ edges $e_{1}, \ldots, e_{k}$ of $\Sigma$, each of length $k \cdot \ell(e)$ mapping down to $e$. Divide each of them into $k$ segments of equal length. For each edge $e_{i}$, we define the slope of $g$ to be $-s$, except for the $i$-th segment, in which the slope is $s \cdot k$. Then $g$ is well defined, continuous, and $\operatorname{div}(g)$ is a divisor mapping down to $E^{\prime}$.

As a result of Proposition 2.7, together with [BF11, Theorem 3.4], the group $J(\Sigma, \Gamma)$ is a real torus of dimension $g_{\Gamma}$. We define the canonical divisor class of $\Gamma$ as $K_{\Gamma}=\phi_{*}\left(K_{\Sigma}\right)$. Note that this is not the same as the canonical divisor of the underlying metric graph of $\Gamma$.

2.5. Theta characteristics and cycles. Accordingly, we define a theta characteristic to be any divisor class $\theta$ satisfying $2 \theta \sim K_{\Gamma}$, which yields a bijection with the 2 -torsion points of the Jacobian $J(\Sigma, \Gamma)$. As a result, $\Gamma$ has $2^{g_{\Gamma}}$ theta characteristics, the same as the cardinality of $H_{0}\left(\Sigma, \mathbb{Z}_{2}\right)$. In [Zha10], Zharkov illustrates a combinatorial recipe for constructing a theta characteristic from every cycle of $\Sigma$. The trivial cycle gives rise to a characteristic with -1 chip at every vertex, and a chip in the midpoint of each edge. This is the non-effective theta characteristic. For a non-trivial cycle $\sigma$, an effective theta characteristic $\theta_{\sigma}$ is constructed as follows. For every point $p$, let $I(p)$ be the number of incoming edges at $p$ from the cycle (in other words, it is the number of edges emanating from $p$ along which the distance from $\sigma$ is decreasing). Then $\theta_{\sigma}=\frac{K_{\Gamma}+\operatorname{div}(I)}{2}$. Explicitly, 
if $p$ is in the cycle, then $\theta_{\sigma}=\frac{\operatorname{val}_{\sigma}(p)-2}{2}$, where $\operatorname{val}_{\sigma}(p)$ is the valency of $p$ restricted to $\sigma$, and $\theta_{\sigma}=\frac{I(p)-1}{2}$ otherwise.

To find the theta characteristics of a plane tropical curve $\Gamma$, we use Zhrakov's algorithm on the paired metric graph $\Sigma$. Whenever two cycles of $\Sigma$ get identified in $\Gamma$, their difference is in the kernel of the pushforward map $\phi_{*}: J(\Sigma) \rightarrow J(\Sigma, \Gamma)$. Consequently, the corresponding theta characteristics map to the same theta characteristic of $\Gamma$.

Example 2.8. Let $\Sigma$ and $\Gamma$ be as in Figure 2. Let $\sigma_{1}$ be the large cycle in the middle of $\Sigma$, and $\sigma_{2}$ the union of the large cycle and the cycle above it. Then $\theta_{\sigma_{1}}=P+R$ and $\theta_{\sigma_{2}}=Q+R$. The images of $\theta_{\sigma_{1}}$ and $\theta_{\sigma_{2}}$ are equivalent divisors in $J(\Sigma, \Gamma)$. This is consistent with the fact that the pairing morphism identifies the cycles $\sigma_{1}$ and $\sigma_{2}$.

Definition 2.9. The multiplicity of a theta characteristic class $\theta$ on a tropical plane curve is the number of effective theta characteristic classes in its preimage $\phi_{*}{ }^{-1}(\theta)$.

Note that $\phi_{*}: J(\Sigma) \rightarrow J(\Sigma, \Gamma)$ is a map of groups. It follows that the fiber of each theta characteristic of $J(\Sigma, \Gamma)$ consists of $2^{g_{\Sigma}-g_{\Gamma}}$ theta characteristics of $\Sigma$. Therefore,

Lemma 2.10. There is a $2^{g_{\Sigma}-g_{\Gamma}}: 1$ map from the set of theta characteristic classes on $\Sigma$ to the set of theta characteristic classes on $\Gamma$. Furthermore, only one among the $2^{g_{\Gamma}}$ theta characteristic classes on $\Gamma$ has $2^{g_{\Sigma}-g_{\Gamma}}-1$ effective theta characteristic classes in its preimage, and the others have $2^{g_{\Sigma}-g_{\Gamma}}$ effective theta characteristic classes in its preimage.

In particular, when $g(\Gamma)=g(\Sigma)$, all the effective theta characteristics of $\Gamma$ have multiplicity 1 . On the other hand, when $g(\Gamma)<g(\Sigma)$, the image of the non-effective theta characteristic is equivalent to an effective divisor. Indeed, it has at least one effective preimage in $\Sigma$, and the pushforward map sends effective divisors to effective divisors.

\section{Bitangent lines to tropical plane Curves}

In this section, we prove that every bitangent to a tropical plane curve gives rise to a theta characteristic. As always $\Gamma$ denotes a tropical plane quartic curve, $\Sigma$ denotes the paired metric graph, and $\phi$ the pairing morphism. Given a tropical line $\Lambda$, we obtain a divisor

$$
\Lambda \cdot \Gamma=\sum_{P \in \Gamma} a_{P} \cdot P
$$

on $\Gamma$, where $a_{P}$ is the multiplicity of the stable intersection of $\Gamma$ and $\Lambda$ at $P$. We remind the reader that to obtain the stable intersection, choose a vector $\mathrm{v}$ such that $\Gamma$ and $\Lambda+\epsilon \cdot v$ intersect properly, and take the limit as $\epsilon$ goes to zero.

A bitangent of $\Gamma$ is, morally speaking, a line that meets the curve with multiplicity at least 2 at two or more points, or at one point with multiplicity at least 4 . However, we need to be a bit more careful when dealing with non-transverse intersections.

Definition 3.1. A tropical line $\Lambda$ is bitangent to $\Gamma$ at $P$ and $Q$ if there exists a piecewise linear function $f$ that is constant outside of the intersection, and such that $\Gamma \cdot \Lambda+\operatorname{Div} f$ is effective and contains $2 P+2 Q$.

For instance, when a component of $\Lambda \cap \Gamma$ is an edge, there is a tangency at the midpoint of the edge.

Lemma 3.2. All the line sections of a plane tropical curve $\Gamma$ are equivalent in $\operatorname{Pic}(\Sigma, \Gamma)$. Their pullbacks are equivalent in $\operatorname{Pic}(\Sigma)$. 
Proof. Let $\Lambda_{1}, \Lambda_{2}$ be tropical lines with defining equations $f_{1}, f_{2}$. For each $i$, consider the function $\psi_{i}$ on $\Sigma$ whose slope at a point $p$ equals the slope of $f_{i}$ at $\phi(p)$. Then $\phi_{*} \operatorname{Div}\left(\psi_{i}\right)=\Lambda_{i} \cdot \Gamma$. Moreover, the two line sections $\Lambda_{1} \cdot \Gamma$ and $\Lambda_{2} \cdot \Gamma$ are equivalent since their difference is the pushforward of the principal divisor $\operatorname{Div}\left(\psi_{2}\right)-\operatorname{Div}\left(\psi_{1}\right)$.

Lemma 3.3. Suppose that a tropical line $\Lambda$ passes through a vertex $u$ of a tropical curve $\Gamma$. Then the stable intersection of $\Gamma$ and $\Lambda$ contains $u$. If the weight $w_{\Gamma}(u)$ is positive, then the stable intersection has multiplicity at least 2 at $u$. Moreover, if the vertex of $\Lambda$ is at $u$, then the stable intersection has multiplicity at least 3 when the weight is 1 , and at least 4 when the weight is greater than 1.

Proof. We begin with the case where $u$ is in the interior of one of the ends of $\Lambda$. Without loss of generality we may assume it is the horizontal end. Then the stable intersection at $u$ equals the width of the polygon dual to $u$ in the Newton subdivision of $\Gamma$. The weight of $u$ equals the number of lattice points in the interior of the polygon. This number can be positive only if the width, and therefore the stable intersection is at least 2 .

Now, assume that $u$ is at the vertex of $\Lambda$. Let $\Gamma_{u}$ be the tropical curve consisting of $u$ and the edges emanating from it (so $\Gamma_{u}$ is the star of $u$ ). The stable intersection of $\Lambda$ with $\Gamma$ at $u$ equals the stable intersection of $\Gamma_{u}$ with a line whose vertex is at $u$. By Bézout's theorem [MS15, Theorem 1.3.2], the degree of the stable intersection equals the degree of $\Gamma_{u}$. If the weight of $u$ is 1 , then this degree at least 3 , and if the weight of $u$ is 2 or more, then this degree is at least 4 .

Proposition 3.4. A line section of a tropical quartic is equivalent to the canonical divisor.

Proof. By Bézout's theorem, $\Lambda \cdot \Gamma$ has degree 4. Consider a divisor $L$ such that $\phi_{*}(L)=\Lambda \cdot \Gamma$. By Riemann-Roch, in order to prove that $L$ is canonical, it suffices to show it has rank 2 . Let $P$ and $Q$ be points of $\Sigma$. By Lemma 3.2, it suffices to find a tropical line whose pullback to $\Sigma$ is equivalent to $P+Q$. Since the vertices of $\Sigma$ are a rank determining set, we can assume that $P$ and $Q$ are either vertices or at the midpoint of loops of $\Sigma$ [Luo11]. Therefore, $\phi(P)$ and $\phi(Q)$ are not in the interior of weighted edges of $\Gamma$. Let $\Lambda$ be a tropical line that passes through $\phi(P)$ and $\phi(Q)$. If $\phi(P)=\phi(Q)$ choose $\Lambda$ to be the line whose vertex is at $\phi(P)$. We distinguish between two cases.

(1) $\phi(P) \neq \phi(Q)$. Let $m=w_{\Gamma}(\phi(P))$, and denote $\gamma=\phi^{-1}(\phi(P))$. Then $\gamma$ is a graph consisting of $m$ loops and a single vertex. By Lemma 3.3 , the pullback of $\Lambda$ to $\gamma$ has degree at least 2 when the weight of $\phi(P)$ is positive, and therefore has rank at least 1 on $\gamma$. In particular, it is equivalent to a divisor containing $P$. A similar argument shows that the pullback of $\Lambda$ to $\phi^{-1}(\phi(Q))$ is equivalent to a divisor containing $Q$.

(2) $\phi(P)=\phi(Q)$. Similarly to the previous case, denote $\gamma=\phi^{-1}(\phi(P))$. Again $\gamma$ is a graph consisting of $m$ loops. This time, since the vertex of $\Lambda$ is at $\phi(P)$, Lemma 3.3 implies that the pullback of $\Lambda$ to $\gamma$ has rank at least 2, and is therefore equivalent to a divisor that contains both $P$ and $Q$.

As an immediate consequence we conclude,

Corollary 3.5. Let $\Lambda$ be a bitangent of a tropical plane quartic $\Gamma$. Then $\frac{\Lambda \cdot \Gamma}{2}$ is a theta characteristic of $\Gamma$.

It is left to show that every effective theta characteristics can be realized as the intersection with a line. We do that in the next section. 


\section{FAMilies of Bitangent Lines}

In this section, we study the behaviour of bitangents of tropical quartics in families, and interpret the multiplicity of the theta characteristics as the number of bitangents converging to it from nearby curves (cf. [CS03, Section 3]).

In order to discuss families of bitangent lines, we set up some notations. The combinatorial type of a weighted metric graph is the finite graph obtained by forgetting the edge lengths. Sequences of plane quartics and their limit are all taken within the moduli space $\mathbb{M}_{g}^{\text {planar }}$ of planar tropical curves of genus $g$ (see [BJMS15]). By Corollary 3.5, every bitangent of a tropical quartic gives rise to a theta characteristic. We use this to define its multiplicity.

Definition 4.1. Two bitangents are equivalent if they correspond to the same theta characteristic. The multiplicity of a bitangency class is defined to be the multiplicity of the theta characteristic.

Said differently, two bitangents are equivalent precisely when we can continuously move one of them to the other, while continuously moving the tangency points.

Example 4.2. Consider the sequence of tropical quartics $\Gamma_{n}$ depicted in Figure 3. The height of the upper rectangle is $\frac{1}{n}$, and the sequence converges to the tropical curve $\Gamma$ on the right side of the figure. For each $\Gamma_{n}$, we consider two bitangent lines $\Lambda_{n}^{\prime}$ and $\Lambda_{n}^{\prime \prime}$. To avoid overcrowding the picture, we only sketched the vertex of each line. Then the two sequences of bitangents converge to $\Lambda$, whose multiplicity according to Definition 4.1 is 2.

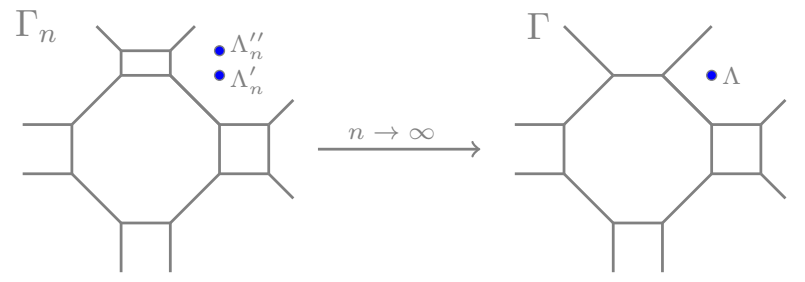

FiguRE 3. A converging sequence of tropical quartics.

Theorem 4.3. Each theta characteristic of $\Gamma$ gives rise to a bitangent line $\Lambda$. Moreover, if $\Gamma_{n}$ is a sequence of tropical quartics converging to $\Gamma$, then the multiplicity of $\Lambda$ equals the sum of the multiplicities of the bitangents of $\Gamma_{n}$ converging to it.

Proof. Let $\Gamma_{n}$ be a squence of tropical curves converging to $\Gamma$. We may assume, perhaps after passing to a subsequence, that their skeletons all have the same combinatorial type $G$, and that the combinatorial type of the curve in the limit is obtained by contracting edges of $G$. Therefore, they all live in the closure of the same cone of $\mathbb{M}_{g}^{\text {planar }}$. Similarly, if we consider the corresponding paired metric graphs $\Sigma_{n}$ and $\Sigma$, we may assume that they all live in the same cone of the moduli space of tropical curves $M_{g}^{\text {Trop }}$, and have the same combinatorial type $H$. We therefore have a canonical identification of the theta characteristics of $\Sigma_{n}$ and $\Sigma$ with cycles of $G$.

Let $\operatorname{Pic}_{2}(H, w)$ be the tropical universal Picard space classifying divisor classes of degree 2 on all the metric graphs of type $H$. Then by [Len14], this space is compact, and the rank of divisors varies upper semicontinuously. In particular, every sequence of theta characteristics has a subsequence that converges to a theta characteristic.

Let $\theta$ be an effective theta characteristic of $\Gamma$ of multiplicity $k$. Then there are $k$ theta characteristics $\theta^{1}, \theta^{2}, \ldots, \theta^{k}$ of $\Sigma$ corresponding to $\Lambda$. Each $\theta^{i}$ is the limit of a sequence of theta characteristics 
$\theta_{n}^{i}$ of $\Sigma_{n}$. By $\left[\mathrm{BLM}^{+} 15\right.$, Theorem 3.9], each of them gives rise to a sequence of bitangent lines $\Lambda_{n}^{i}$ of $\Gamma_{n}$, which converges to a bitangent of $\Gamma$. Note that the lines $\Lambda_{n}^{i}$ are in fact in distinct bitangency classes of $\Gamma_{n}$ since they correspond to distinct theta characteristics. Therefore the multiplicity of $\Lambda$ equals the sum of the multiplicities of the bitangents of $\Gamma_{n}$ converging to it.

We are now ready to prove Theorem 4.4 .

Theorem 4.4. Let $\Gamma$ be a tropical plane quartic curve with genus $g$. If $g$ is 3 , it has 7 equivalence classes of bitangent lines each with multiplicity 1. If $g$ is less than 3 , it has $2^{g}$ equivalence classes of bitangent lines: only one of them has multiplicity $2^{3-g}-1$, and all the others have multiplicity $2^{3-g}$.

Proof. Let $\Gamma$ be a tropical quartic. By Lemma 2.10, it has one effective theta characteristic of multiplicity $2^{3-g}-1$, and all others have multiplicity $2^{3-g}$. By Theorem 4.3 , each of them corresponds to a bitangent line whose multiplicity equals the multiplicity of the corresponding theta characteristic.

Remark 4.5. Most of the ingredients of the proof of $\left[\mathrm{BLM}^{+} 15\right.$, Theorem 3.9] carry well to the nonsmooth case. Therefore, our Theorem 4.4 could also be proven directly, by making the necessary adjustments.

We finish by asking about the relation between algebraic and tropical bitangents to quartics. When $\Gamma$ is a smooth quartic, there are 4 bitangents of $C$ tropicalizing to every bitangency class of $\Gamma$ [JL16, LM17].

Conjecture 1. Let $C$ be an algebraic plane quartic curve over the field of Puiseux series, and let $\Gamma=\operatorname{Trop}(C)$. Let $[\Lambda]$ be an equivalence class of bitangents of $\Gamma$ of total multiplicity $m$. Then there are $4 m$ bitangents of $C$ tropicalizing to $[\Lambda]$.

\section{REFERENCES}

[BF11] M. Baker and X. Faber. Metric properties of the tropical Abel-Jacobi map. J. of Alg. Comb., 33(3):349-81, 2011.

[BJ16] M. Baker and D. Jensen. Degeneration of Linear Series from the Tropical Point of View and Applications, pages 365-433. Springer International Publishing, Cham, 2016.

[BJMS15] S. Brodsky, M. Joswig, R. Morrison, and B. Sturmfels. Moduli of tropical plane curves. Res. Math. Sci., 2:Art. 4, 31, 2015.

$\left[\mathrm{BLM}^{+} 15\right]$ M. Baker, Y. Len, R. Morrison, N. Pflueger, and Q. Ren. Bitangents of tropical plane quartic curves. Mathematische Zeitschrift, pages 1-15, 2015.

[CJ15] M. Chan and P. Jiradilok. Theta characteristics of tropical $k_{4}$-curves. Preprint arXiv:1503.05776, 2015.

[CS03] L. Caporaso and E. Sernesi. Recovering plane curves from their bitangents. J. Algebraic Geom., 12(2):225244, 2003.

[GK08] A. Gathmann and M. Kerber. A Riemann-Roch theorem in tropical geometry. Mathematische Zeitschrift, 259:217-230, 2008.

[JL16] D. Jensen and Y. Len. Tropicalization of theta characteristics, double covers, and Prym varieties. Preprint arXiv:1606.02282, June 2016.

[Len14] Y. Len. The Brill-Noether rank of a tropical curve. J. Algebraic Combin., 40(3):841-860, 2014.

[LM17] Y. Len and H. Markwig. Lifting tropical bitangents. Preprint arXiv:1708.04480, August 2017.

[Luo11] Y. Luo. Rank-determining sets of metric graphs. J. Comb. Theory Series A, 118(6):1775-1793, 2011.

[MS15] D. Maclagan and B. Sturmfels. Introduction to tropical geometry. Providence, RI: American Mathematical Society (AMS), 2015.

[Pan15] M. Panizzut. Theta characteristics of hyperelliptic graphs. Preprint arXiv:1511.07243, 2015.

[Pic99] G. Pick. Geometrisches zur Zahlenlehre. Sonderabdr. Naturw.-medizin. Verein f. Böhmen "Lotos" Nr. 8, 9 S. $8^{\circ}, 1899$.

[Zha10] I. Zharkov. Tropical theta characteristics. In Mirror symmetry and tropical geometry, volume 527 of Contemp. Math., pages 165-168. Amer. Math. Soc., Providence, RI, 2010. 